ORIGINAL ARTICLE

\title{
Early physiological development of infants with intrauterine growth retardation
}

\author{
J A Jackson, M P Wailoo, J R Thompson, S A Petersen
}

Arch Dis Child Fetal Neonatal Ed 2004;89:F46-F50

See end of article for authors' affiliations

.....................

Correspondence to: M P Wailoo, Department of Child Health, University of Leicester, PO Box 65, Leicester LE1 7LX, UK; mw33@le.ac.uk

Accepted 21 January 2003
Objectives: To assess the patterns of early postnatal physiological adaptation and maturation in intrauterine growth retarded (IUGR) infants by measuring changes in sleeping deep body temperature, heart rate, and concentrations of urinary cortisol.

Setting: At home.

Patients: Sixty five IUGR babies and 127 controls matched for sex, social class, and levels of parental smoking.

Results: Night time sleeping deep body temperature, heart rate, and cortisol excretion fell with age, eventually establishing an adult type diurnal rhythm of physiological function. Minimum overnight temperature showed a linear decline with age $(p<0.001)$, but the IUGR infants and the controls had significant differences in intercept $(p=0.007)$ and slope $(p=0.02)$. The estimated rate of decline per week was $0.020^{\circ} \mathrm{C}$ for IUGR infants and $0.031^{\circ} \mathrm{C}$ for controls. Maximum temperature did not show similar changes. IUGR infants had a mean (SE) age adjusted minimum overnight heart rate that was 4.2 (1.5) beats $/ \mathrm{min}(p=0.005)$ higher than controls. Overnight cortisol/creatinine ratios declined with age at a rate of $4.1 \%$ per week ( $\log$ ratio $-0.421(0.0165), p=0.01)$, but the ratio for IUGR infants was on average $42 \%$ higher (log ratio $0.35(0.11), p=0.002$ ) than for controls of the same age. Morning cortisol concentrations did not show a similar pattern.

Conclusions: Postnatal physiological adaptation and maturation of IUGR infants is slower than normal and therefore they remain in a physiologically immature state for longer. The higher heart rates and greater cortisol excretion in such infants may be precursors to hypertension and cardiovascular disease seen in adults.
$\mathrm{N}$ ot only do antenatal events dictate the pattern of infant mortality and morbidity, but there is growing evidence that they may be associated with illness in later life. Hypertension, coronary heart and cardiovascular disease, and diabetes appear to be more likely in adults who were growth retarded infants. ${ }^{1-3}$

Intrauterine growth retarded (IUGR) infants have more fatal congenital deformities and are therefore more likely to die neonatally. There is also more illness in infancy ${ }^{4}$ and more sudden unexplained infant deaths. ${ }^{5}$

Factors associated with intrauterine growth retardation vary from poor maternal health and substandard nutrition to maternal drug taking and cigarette smoking, ${ }^{6}$ but the continuing mechanism by which the lasting effects of an antenatal insult can be transmitted into adult life is unknown. Phillips et $\mathrm{al}^{7}$ suggested that raised levels of plasma cortisol in adults who were initially growth retarded infants may be a contributory factor to the development of hypertension and insulin dependent diabetes. We have previously studied the way babies adapt to their new environment after birth by evaluating physiological variables such as age related changes in deep body temperature with sleep, heart rate, and cortisol excretion ${ }^{8-10}$ in normal and other children in whom the rate of adaptation varies widely. In this study we report on the way similar changes occur in growth retarded infants, focusing on age 6-20 weeks, the period of maximum physiological change which coincides with the period of maximum infant mortality and morbidity.

\section{METHODS}

Full perinatal data were collected on 65 infants who were identified as having suffered IUGR either by serial ultrasound scans, which identified infants who had fetal abdominal circumference more than two standard deviations below the mean, or by birth weights on or below the 2nd centile for gestational age. ${ }^{11}$ Parental consent was obtained to monitor each infant in repeated visits to their home. At each visit, the infant was weighed naked. Probes were attached to record body temperature and heart rate. Uri bags were attached to the perineal area to collect overnight and morning samples of urine.

A soft probe was inserted $5 \mathrm{~cm}$ into the rectum to record deep body temperature, a skin thermistor measured the surface of the skin, and a third probe measured the ambient temperature of the room in which the infant slept. These probes were attached to a Grant Squirrel data logger which was set to record the temperatures simultaneously at one ?minute intervals throughout the night, from the time the baby was put to bed to the time it awoke the next morning. Heart rate was measured using three disposable chest leads positioned in the standard limb configuration and an oxiband oxygen transducer attached around the infant's foot using a disposable adhesive strap to maintain the correct position. These leads were then attached to a Drager Nellcor Oxymeter N-200E positioned next to the sleeping infant. The oximeter sampled every second and averaged every five seconds of readings taken.

Whenever possible, two urine specimens were collected using a paediatric 24 hour Uri bag attached to a collection bottle and left on free drainage. During the evening, the Uri bag was attached to the infant as described by the manufacturers. A second Uri bag was used to collect the morning specimen of urine. Urine samples were deep frozen within four hours and subsequently analysed for creatinine by standard techniques and for cortisol by extractive radio- 
immunoassay (Coat a Count assay). ${ }^{12}$ All these techniques are safe, and full ethical approval was obtained for the study.

Parents were asked to keep a detailed diary of all events that occurred on the night of the recording, including nappy changes, periods of waking and feeding, and any medication given to the infant. The following morning, the equipment was collected and the recorded measurements were downloaded to a computer for analysis. The recordings were scrutinised for evidence of lost probes or technical problems, and affected data were removed before further analysis. For each night, measurements were abstracted at 30 minute intervals, the rectal temperature for the period from one hour before bedtime until eight hours later, and the heart rate for the period from bedtime until six hours later.

For each IUGR infant, data on matched control infants who had identical physiological measurements were taken from an existing database. The IUGR infants and controls were individually matched for sex, social class, method of feeding, and maternal smoking behaviour.

The measurements were analysed by mixed normal regression models fitted by REML. ${ }^{13}$ The models incorporated a random subject term to allow for the correlation between repeated measures on the same infant. Cortisol/creatinine ratios were positively skewed and so the log of the ratios was analysed.

\section{RESULTS}

Sixty five infants with IUGR were identified. They were monitored for temperature, heart rate, and cortisol/creatinine ratio on an average of 4.2 occasions between the ages of 6 weeks and 21 weeks. Matched controls were chosen from a large database of healthy children who had been studied previously using the same methodology.

\section{Temperature}

The analysis included 64 IUGR infants and 127 controls. Overnight rectal measurements were discarded when the probe became loose, when there were missing data in the first four hours after bedtime, or when the infant was monitored on a night after an immunisation. On average, each IUGR infant was successfully monitored 4.0 times, and each control infant 3.5 times between the ages of 6 weeks and 21 weeks, providing a total of 712 temperature recordings, 258 for the IUGR infants and 454 for the controls. Figure 1 shows the ages at which the IUGR infants and their controls were monitored. Most monitoring took place between 7 and 15 weeks of age.

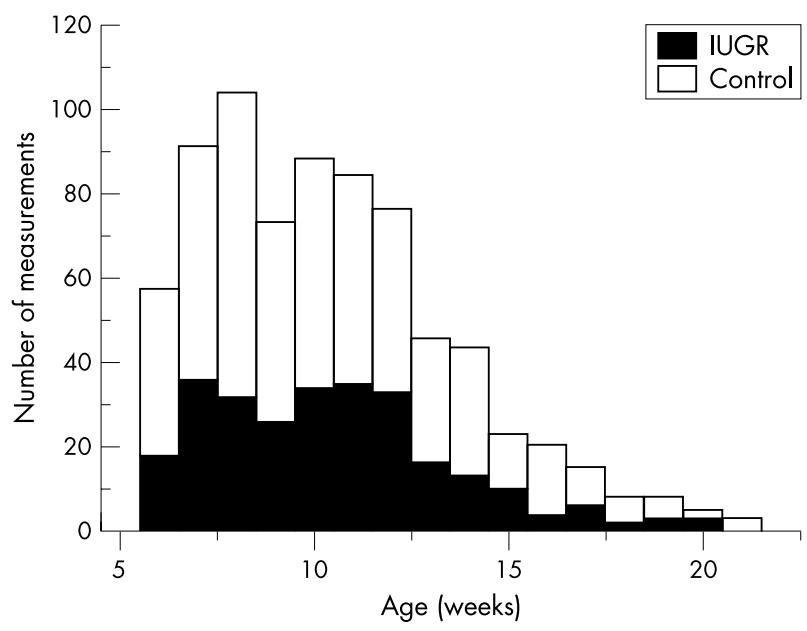

Figure 1 Age at time of monitoring. Most infants were monitored at 615 weeks of age. IUGR, Intrauterine growth retarded.
Table 1 Comparison of matching and other variables: temperature analysis

\begin{tabular}{lll}
\hline & IUGR ( $\mathbf{n = 6 4 )}$ & Controls ( $\mathbf{n}=127)$ \\
\hline Matched factors & & \\
Sex (male) & $46 \%$ & $44 \%$ \\
Feeding (breast) & $48 \%$ & $45 \%$ \\
$\quad$ Maternal smoking (yes) & $54 \%$ & $47 \%$ \\
Social class & & \\
$\quad$ I or II & $21 \%$ & $30 \%$ \\
$\quad$ IIIn or IIIm & $52 \%$ & $46 \%$ \\
$\quad$ IV or less & $29 \%$ & $24 \%$ \\
Unmatched factors & & \\
Maternal age (years) & $27.8(5.0)$ & $28.6(4.8)$ \\
$\quad$ Gestation (weeks) & $38.7(1.5)$ & $39.8(1.5)$ \\
Birth weight (g) & $2663(453)$ & $3448(506)$ \\
\hline
\end{tabular}

Values are mean (SD) or percentages.

IUGR, Intrauterine growth retarded; IIIn, social class III, non-manual; IIIm, social class III, manual.

Table 1 shows the quality of the matching. The mothers of controls tended to be of slightly higher social class and to be marginally more likely to be non-smokers. Counting the number of variables by which the IUGR infants and their matched controls differed and treating social classes that differ by more than one category as being a mismatch, the average difference between a control and their IUGR infant was 0.5 of a variable.

Temperatures were extracted from the continuous recording at 30 minute intervals between bedtime and four hours after bedtime. From the resulting nine temperature readings for a given night, the maximum and minimum temperatures were calculated. Figure 2 shows the means of the minimum and maximum temperatures across all IUGR infants and across their controls.

Models for the minimum temperature showed a linear decline $(p<0.001)$ and no significant deviation from linearity $(p=0.57)$. The lines for IUGR infants and controls showed significant differences in intercept $(p=0.007)$ and slope $(\mathrm{p}=0.02)$. The intercepts differed by a mean (SE) of $0.051(0.019){ }^{\circ} \mathrm{C}$, and the estimated decline for IUGR infants was $0.020(0.004){ }^{\circ} \mathrm{C}$ a week whereas that for the controls was $0.011(0.005)^{\circ} \mathrm{C}$ a week steeper. Patterns of linear trend require careful interpretation and they sometimes show good local fit when the model does not extrapolate. ${ }^{14}$ In this study an alternative model for the data would be that IUGR infants are similar to controls until about 10 weeks. They then show

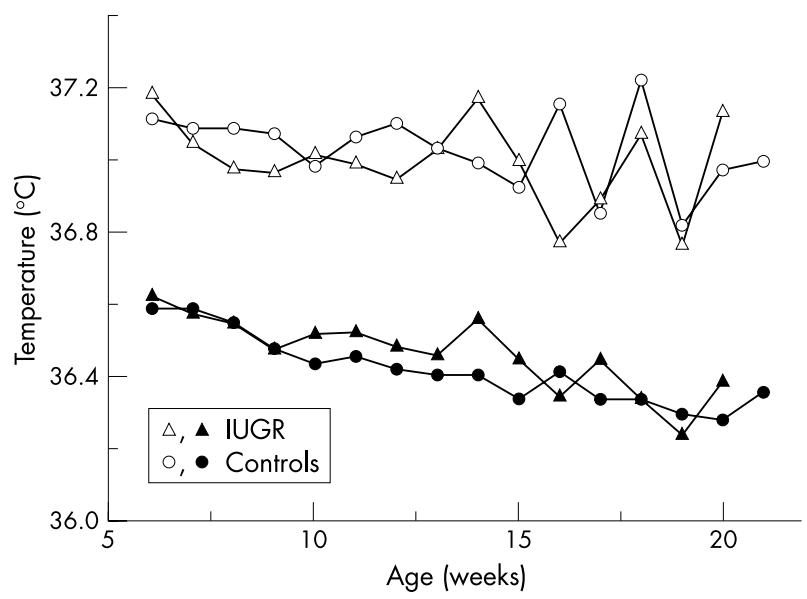

Figure 2 Maximum (open symbols) and minimum (closed symbols) night time body temperatures of intrauterine growth retarded (IUGR) and control infants. 
Table 2 Comparison of matching and other variables: heart rate analysis

\begin{tabular}{lll}
\hline & IUGR ( $\mathbf{n}$ 54) & Controls ( $\mathbf{~ = ~ 5 4 ) ~}$ \\
\hline Matched factors & & \\
Sex (male) & $59 \%$ & $52 \%$ \\
Feeding (breast) & $50 \%$ & $31 \%$ \\
Maternal smoking (yes) & $50 \%$ & $59 \%$ \\
Social class & & \\
$\quad$ I or II & $17 \%$ & $15 \%$ \\
$\quad$ IIIn or IIIm & $57 \%$ & $37 \%$ \\
$\quad$ IV or less & $26 \%$ & $48 \%$ \\
Unmatched factors & & \\
$\quad$ Maternal age (years) & $27.5(5.1)$ & $26.9(4.4)$ \\
Gestation (weeks) & $38.7(1.5)$ & $39.8(1.5)$ \\
Birth weight (g) & $2633(405)$ & $3328(462)$ \\
\hline & \\
Values are mean (SD) or percentages. \\
IUGR, Intrauterine growth retarded.
\end{tabular}

less of a decline in minimum temperature but catch up about a month later. A post hoc definition of the 10-15 week interval as being where the groups differ produces a significant interaction $(p=0.02)$ with a mean difference between groups within those ages of $0.092^{\circ} \mathrm{C}$, but not otherwise. As this interval is derived from these data, the finding requires confirmation in another study.

The maximum temperatures showed a declining trend $(p=0.003)$ but there was also a suggestion of non-linearity $(p=0.02)$. Adjusting for the age difference between IUGR infants and controls was of marginal significance $(p=0.07)$, with the maximums for the IUGR infants on average 0.44 $(0.024){ }^{\circ} \mathrm{C}$ lower than for the controls. There was no suggestion of a difference in slope $(\mathrm{p}=0.53)$ or of a difference between 10 and 15 weeks that was absent elsewhere $(p=0.48)$.

\section{Heart rate}

All of the 54 IUGR infants were matched with a control. Table 2 shows the quality of the matching. The control infants for whom heart rate was available tended to come from poorer social backgrounds $(\mathrm{p}=0.06)$ and to be less likely to have been breast fed $(\mathrm{p}=0.05)$. Counting mismatches in the four variables that were used for matches, on average the IUGR infant and their control differed by 0.9 of a variable.

The 54 IUGR infants included in the heart rate analysis had been monitored successfully over 211 nights, and the 54 controls on 142 nights. Half hourly readings were extracted

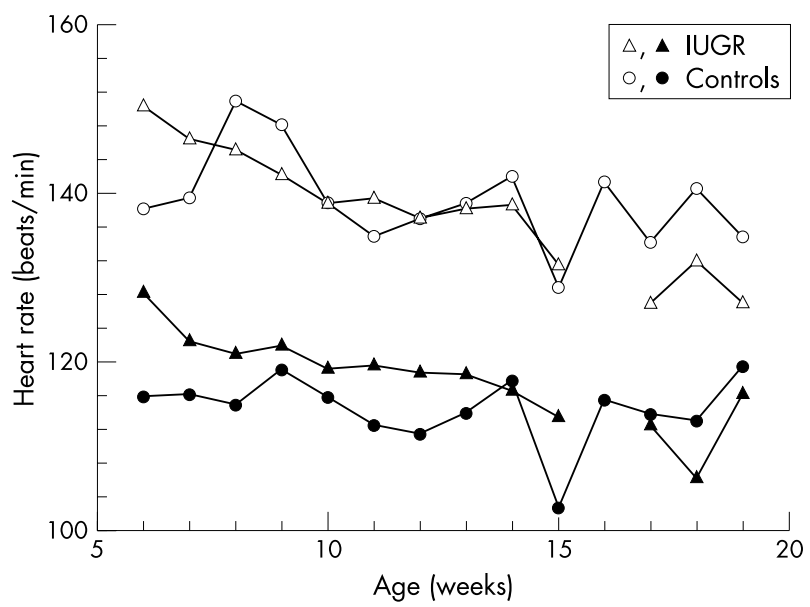

Figure 3 Maximum (open symbols) and minimum (closed symbols) heart rate in intrauterine growth retarded (IUGR) and control infants.
Table 3 Comparison of matching and other variables: bedtime cortisol to creatinine ratio

\begin{tabular}{|c|c|c|}
\hline & IUGR ( $n=39$ ) & Controls $(n=58)$ \\
\hline \multicolumn{3}{|l|}{ Matched factors } \\
\hline Sex (male) & $62 \%$ & $60 \%$ \\
\hline Feeding (breast) & $59 \%$ & $50 \%$ \\
\hline Maternal smoking (yes) & $44 \%$ & $34 \%$ \\
\hline \multicolumn{3}{|l|}{ Social class } \\
\hline | or || & $31 \%$ & $40 \%$ \\
\hline IIIn or IIIm & $49 \%$ & $51 \%$ \\
\hline IV or less & $21 \%$ & $9 \%$ \\
\hline \multicolumn{3}{|l|}{ Unmatched factors } \\
\hline Maternal age (years) & $28.3(5.3)$ & $30.0(4.2)$ \\
\hline Gestation (weeks) & $38.4(1.4)$ & $40.2(1.6)$ \\
\hline Birth weight $(\mathrm{g})$ & $2642(491)$ & $3552(494)$ \\
\hline
\end{tabular}

from the continuous recordings taken over the four hours after bedtime, and the minimum and maximum of these values were calculated. Figure 3 shows the means.

The analysis of minimum heart rate showed a decline with age averaging $1.0(0.2)$ beats/min a week. Adjusting for age, the IUGR infants had a higher minimum heart rate by a mean (SE) of $4.2(1.5)$ beats/min $(p=0.005)$. There was an inconclusive suggestion of a difference in slope between IUGR infants and controls $(p=0.07)$, but no evidence of a different pattern in weeks 10-15 compared with other ages $(\mathrm{p}=0.65)$. The maximum heart rates also showed a decline with age, which in this case averaged $1.5(0.22)$ beats/min a week, but there was no suggestion of a difference between IUGR infants and controls $(\mathrm{p}=0.22)$.

To compensate for the poor matching of social class, the minimum heart rate was reanalysed including a term for social class based on the groupings shown in table 2 . The rate of change with age stayed at $1.0(0.2)$ beat/min a week, and the mean age adjusted difference between IUGR infants and controls increased to 4.9 (1.7) beats/min ( $p=0.005)$.

\section{Cortisol}

Cortisol and creatinine concentrations were measured in urine samples collected overnight and again in the morning. Cortisol/creatinine ratios were then calculated. Two controls were sought for each IUGR infant.

\section{Overnight cortisol}

Thirty nine of the IUGR infants had at least one bedtime measurement and could be matched to at least one control with a bedtime measurement. Cortisol was measured in all the 39 IUGR infants on 114 occasions and in the 58 matched controls on 160 occasions. Table 3 shows a summary of the quality of the matching.

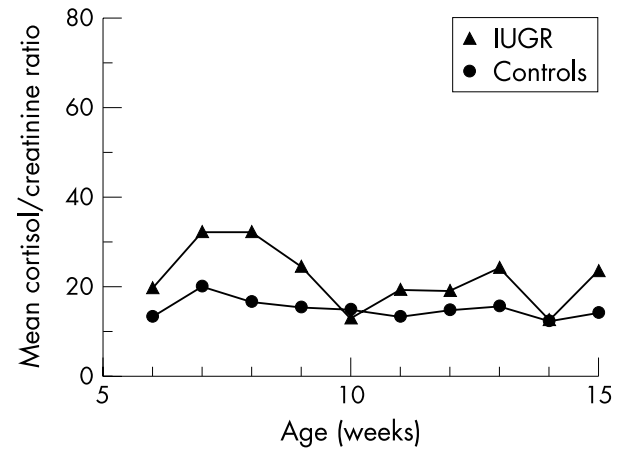

Figure 4 Bedtime cortisol/creatinine ratios in urine of intrauterine growth retarded (IUGR) and control infants. 


\begin{tabular}{|c|c|c|}
\hline & IUGR $(n=31)$ & Controls $(n=44)$ \\
\hline \multicolumn{3}{|l|}{ Matched factors } \\
\hline Sex (male) & $65 \%$ & $66 \%$ \\
\hline Feeding (breast) & $55 \%$ & $48 \%$ \\
\hline Maternal smoking (yes) & $39 \%$ & $32 \%$ \\
\hline \multicolumn{3}{|l|}{ Social class } \\
\hline I or II & $39 \%$ & $39 \%$ \\
\hline IIIn or IIIm & $35 \%$ & $52 \%$ \\
\hline IV or less & $26 \%$ & $9 \%$ \\
\hline \multicolumn{3}{|l|}{ Unmatched factors } \\
\hline Maternal age (years) & $39.0(5.5)$ & $30.7(4.2)$ \\
\hline Gestation (weeks) & $38.5(1.4)$ & $40.0(1.7)$ \\
\hline Birth weight (g) & $2694(520)$ & $3568(501)$ \\
\hline
\end{tabular}

Figure 4 shows the average bedtime cortisol/creatinine ratios. On analysis of the $\log$ of the ratio, there was a suggestion of a decline with age of $4.1 \%$ a week (log ratio $=-0.0421, \mathrm{SE}=0.0165, \mathrm{p}=0.01$ ) and strong evidence that IUGR infants had higher cortisol/creatinine ratios. The IUGR infants had ratios that were $42 \%$ higher (log ratio $=0.35, \mathrm{SE} 0.11, \mathrm{p}=0.002$ ) than the controls. There is no suggestion of a difference in the rate of decline with age depending on the IUGR status of the child $(\mathrm{p}=0.92)$.

\section{Morning cortisol}

Thirty one of the IUGR infants had at least one morning measurement and could be matched to at least one control with a morning measurement. Cortisol was measured in all 31 IUGR infants on 85 occasions and in the 44 matched controls on 104 occasions. Table 4 shows a summary of the quality of the matching.

The mean morning cortisol/creatinine ratios (fig 5) were far more variable than the bedtime ratios. There was no suggestion of a decline with age $(p=0.80)$, but there was evidence that IUGR infants have higher cortisol/creatinine ratios (estimated to be $48 \%$ higher; log ratio $=0.391$, SE $0.116, \mathrm{p}<0.001)$.

\section{DISCUSSION}

On the face of it, growth restricted infants appear to be physiologically different during the first postnatal months from other infants. The changes we have measured (the slowing of heart rate, the fall in deep body temperature with sleep, and the excretion of cortisol in the urine) are definitely related to the growth restriction per se and not to some other shared confounding factors identified by Lodemore et $a l^{15}$ and Tuffnell et al. ${ }^{16}$

Noticeably, the changes occur during night time sleep, and the longitudinal nature of this study shows an age related link; together they point to a developmental process during which physiological maturation and adaptation occur leading to the establishment of full adult type circadian rhythms. Presumably, all physiological systems are integrated into a 24 hour cycle influenced by light, darkness, and sleep. ${ }^{17}$ Our findings, which are assumed to be related to sleep although sleep staging was not assessed, show that circadian rhythmicity is established more slowly in growth restricted infants than in "normal" children. This is in keeping with findings of other studies ${ }^{18}$ and suggests that growth restricted infants are slower in adapting and maturing physiologically, and therefore remain in an immature physiological state for longer than other infants. Whether the faster heart rate and the higher cortisol concentration are stressful to the cardiovas-

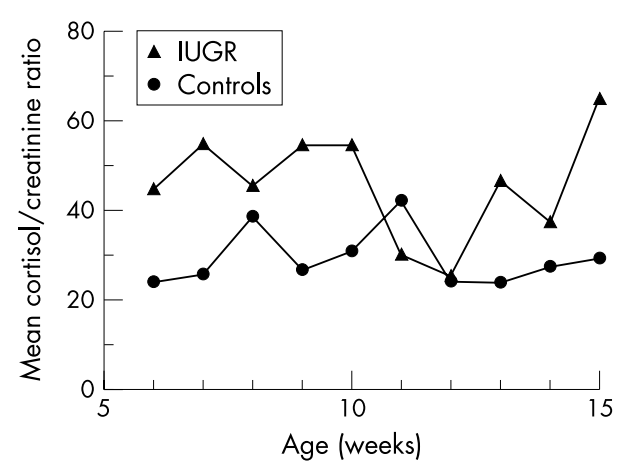

Figure 5 Morning cortisol/creatinine ratios in urine of intrauterine growth retarded (IUGR) and control infants.

cular system, and may therefore be precursors of problems later in life, remains unproven.

The mechanism by which these changes take place is obscure; but the age related nature of the alterations, their apparent connection with sleep, and the integration of multisystem change all point to a central system of control, probably regulated neurohumourly and genetically. Measuring urine cortisol avoids the ethical and practical difficulties of using plasma measurements. Doerr et $a l^{19}$ carried out such studies, albeit on younger growth restricted infants. They found lower glucocorticoid concentrations immediately after birth, but higher than normal concentrations after 24 hours. This is similar to the findings here, and may be the first indication of physiological maturation and adaptation.

Urinary cortisol measurement expressed as a ratio of creatinine may be subject to fluctuations in renal clearance and muscle breakdown. There is no reason to assume that differences in cortisol/creatinine ratios would be accounted for by lower creatinine levels in growth restricted infants. The longitudinal nature of this study allows a clear age related process to be described, and the proportionate changes seen in heart rate, cortisol excretion, and deep body temperature suggest that the changes are part of a wider and perhaps general integrated physiological change. Excretion of melatonin, also rhythmically and age related, is delayed in growth restricted infants ${ }^{20}$ in a way that fits with the findings of this study.

There is every likelihood that IUGR infants are subjected to some intrauterine stress and programming, ${ }^{21}$ which may be compounded by postnatal infant and parental behaviours. When awake, IUGR infants tend to be more passive, difficult to arouse, and prone to more frequent changes of waking state, $^{22}$ and, when asleep, they change states more frequently. ${ }^{23}$ These differences may persist for at least six months ${ }^{24}$ and may be visible expressions of the altering physiological state of the infants. It has been found that the mothers of IUGR babies may be more irritable and less affectionate than normal towards their babies in the first few months. ${ }^{25}$ This raises the question of whether such differences can affect the changes in deep body temperature, heart rate, and cortisol.

The pattern of adult cortisol secretion, diurnal with relatively low night time and high waking-morning concentrations, is slowly achieved by most infants, but more slowly by IUGR infants, who continued to excrete more cortisol than controls up to the time when measurements ceased in this study. In view of the assertions of Barker $e t a l^{2}$ that adults who were growth restricted infants tend to develop more cardiovascular and hypotensive illness, and may also have persistently higher serum concentrations of cortisol, ${ }^{7}$ we speculate that the early physiological changes seen in this 
study may well be the result of early fetal programming which persists into adulthood.

\section{ACKNOWLEDGEMENTS}

We thank Babes in Arms, the Foundation for the Study of Infant Deaths and the Scottish Cot Death Trust for support.

\section{Authors' affiliations}

J A Jackson, Department of Child Health, University of Leicester and University of Warwick, UK

M P Wailoo, Department of Child Health, University of Leicester

J R Thompson, Department of Ophthalmology, University of Leicester

S A Petersen, Department of Medical Education, University of Leicester

\section{REFERENCES}

1 Hales CN, Barker DJ, Clark PM, et al. Fetal and infant growth and impaired glucose tolerance at age 64. BMJ 1991;303:1019-22.

2 Barker DJ, Martyn CN, Osmond C, et al. Growth in utero and serum cholesterol concentrations in adult life. BMJ 1993;307:1524-7.

3 Barker DJ. Fetal origins of coronary heart disease. BMJ 1995;311:171-4.

4 Seeds JW. Impaired fetal growth: definition and clinical diagnosis. Obstet Gynecol 1984;64:303-10.

5 Buck GM, Cookfair DL, Michalek AM, et al. Intrauterine growth retardation and risk of sudden infant death syndrome (SIDS). Am J Epidemiol 1989;129:874-84.

6 Pollack RN, Divon MY. Intrauterine growth retardation: definition, classification and etiology. Clin Obstet Gynecol 1992:35:99-107.

7 Phillips DI, Barker DJ, Fall CH, et al. Elevated plasma cortisol concentrations: a link between low birthweight and the insulin resistance syndrome? J Clin Endocrinol Metab 1998;83:757-60.

8 Lodemore M, Petersen SA, Wailoo MP. Development of night time temperature rhythms over the first six months of life. Arch Dis Child $1991 ; 66: 521-4$.
9 Tappin DM, Ford RP, Nelson KP, et al. Breathing, sleep state and rectal temperature oscillations. Arch Dis Child 1996;74:427-31

10 Wailoo MP, Petersen SA. Relationship between daily rhythms of rectal temperatures and cortisol excretion in human infants. J Physiol 1994:47:83.

11 United Kingdom Growth Charts 1996. Child growth. London, 1996.

12 Murphy BE. How much "UFC" is really cortisol? Clin Chem 2000;46:793-4.

13 Genstat Committee. Genstat 5, release 3 reference manual. Oxford: Oxford University Press, 1993

14 Maclure M, Greenland S. Tests for trend and dose response: misinterpretations and alternatives. Am J Epidemiol 1992;135:96-104.

15 Lodemore MR, Petersen SA, Wailoo MP. Factors affecting the development of night time temperature rhythms. Arch Dis Child 1992;67:1259-61.

16 Tuffnell CS, Petersen SA, Wailoo MP. Factors affecting rectal temperature in infancy. Arch Dis Child 1995;73:443-6.

17 Rappert SM, Weaver DR. Co-ordination of circadian timing in mammals. Nature 2002;48:935-41.

18 Kennaway DJ. Factors influencing melatonin rhythmicity in humans. J Clin Endocrinol Metab 2000;81:1525-31.

19 Doerr HG, Versmold HT, Bidlingmaier F, et al. Adrenocorticoid steroids in small-for-gestational-age term infants during the early neonatal period. Pediatr Res 1989;25:115-18.

20 Kennaway DJ, Flanagan DE, Moore VM, et al. The impact of fetal size and length of gestation on 6-sulphatoxymelatonin excretion in adult life. J Pineal Res 2001;30:188-92.

21 Langley-Evans SC. Intrauterine programming of hypertension by glucocorticoids. Life Sci 1997;60:1213-21.

22 Watt J, Strongman KT. Mother-infant interactions at 2 and 3 months in preterm small-for-gestational-age and full-term infants; their relationship with cognitive development at 4 months. Early Hum Dev $1985 ; 11: 231-46$.

23 Watt JE, Strongman KT. The organization and stability of sleep states in fullterm, preterm, and small-for-gestational-age infants: a comparative study. Dev Psychobiol 1985; 18:151-62.

24 Watt J. Interaction and development in the first year II. The effects of intrauterine growth retardation. Early Hum Dev 1986;13:21 1-23.

25 Pryor J. The identification and long term effects of fetal growth restriction. Br J Obstet Gynaecol 1997; 104:11 16-22.

You can access the FULL TEXT of articles cited in Archives of Disease in Childhood online if the citation is to one of the more than 200 journals hosted by HighWire (http://highwire.stanford.edu) without a subscription to that journal. There are also direct links from references to the Medline abstract for other titles. 